\title{
The Galactic halo stellar density distribution from photometric survey data: results of a pilot study
}

\author{
M. Cignoni ${ }^{1}$, V. Ripepi ${ }^{1}$, M. Marconi ${ }^{1}$, J. M. Alcalá ${ }^{1}$, M. Capaccioli $^{1}$, M. Pannella ${ }^{2}$, and R. Silvotti ${ }^{1}$ \\ 1 Osservatorio Astronomico Di Capodimonte, via Moiariello 16, 80131 Napoli, Italy \\ e-mail: cignoni@df.unipi.it \\ ${ }^{2}$ Max-Planck-Institut für extraterrestrische Physik, Giessenbachstrasse, Postfach 1312, 85741 Garching, Germany
}

Received 19 October 2006 / Accepted 6 November 2006

ABSTRACT

\begin{abstract}
Aims. Our goal is to recover the Galactic halo spatial density by means of field stars. To this aim, we have applied a new technique to the Capodimonte Deep Field (OACDF, Alcalá et al. 2004), as a pilot study in view of the VLT Survey Telescope (VST) stellar projects. Considering this unique chance to collect deep and wide-field photometry with the VST, our method may represent a useful tool towards definitive mapping of the Galactic halo.

Methods. In the framework of synthetic stellar populations, turn-off stars are used to reconstruct the spatial density. The space density is determined by comparing the data with synthetic color-magnitude diagrams (CMDs). The only assumptions involve the IMF, age, and metallicity of the synthetic halo population. Stars are randomly placed in the solid angle. The contributions of the various Monte Carlo distributions (with a step of $4 \mathrm{kpc}$ ) along the line of sight are simultaneously varied to reproduce the observed CMD. Results. Our result for the space density is consistent with a power-law exponent $n \approx 3$ over a range of Galactocentric distances from 8 to $40 \mathrm{kpc}$
\end{abstract}

Key words. Galaxy: halo - Galaxy: structure - Galaxy: stellar content - stars: Hertzsprung-Russell (HR) and C-M diagrams methods: statistical

\section{Introduction}

The astrophysical interest in the spatial distribution of the Galactic halo is fueled by many important questions concerning the origin of our Galaxy and its cosmological implications. In the classical scenario (Eggen et al. 1962) the origin of the halo is identified in a rapid collapse of a proto-galactic cloud. An alternative scenario (Searle \& Zinn 1978) suggests a different history with the Galaxy (and the halo) built from smaller blocks, where the stars have already started developing. Signs of the accretion process are often identified in the observed streams of gas and stars, remnants of tidally-disrupted satellite galaxies. The emerging picture, suggested by recent results, has hybrid nature: the outer halo may have been formed from the accretion of satellite galaxies, while the inner halo is thought to be a relic of a dissipative collapse. One piece of evidence in favor of this scenario is that the axis ratio seems to vary from inner to outer regions (see e.g. Sommer-Larsen \& Zhen 1990; Gould et al. 1998). Variations of the $[\alpha / \mathrm{Fe}]$ ratio abundance are also observed: stars with lower $[\alpha / \mathrm{Fe}]$ tend to stay at larger orbits than the richer ones, suggesting that the outer parts of the halo may have been accreted by intruder galaxies (see e.g. Nissen \& Schuster 1997).

In this context, the observation of a particular decline in the density slope could help for identifying the correct scenario. The mass-density profile has been extensively studied, both using $N$-body simulations and matching suitable stellar tracers. Different power law indexes $n\left(r^{-n}\right)$ are found:

$-n \approx 2$ is required to explain the flat rotation curve;

- $n$ between 2.5 to 3.8 (e.g. Avila-Reese et al. 1999) is supported by $\mathrm{N}$-body simulations for outer halos; $-n \approx 3$ is found from satellite galaxies (SDSS data) orbiting isolated galaxies (Prada et al. 2003);

- $n \approx 3.3$ is found from local halo stars (Sommer-Larsen \& Zhen 1990) in the galactocentric interval 8-20 kpc;

- $n \approx 3$ is derived from RR Lyrae stars (Vivas \& Zinn 2003; Ivezic et al. 2004) in the galactocentric range 4-60 kpc;

$-n \approx 2.7$ (Siegel et al. 2002) and $n \approx 2.5$ (Robin et al. 2000) from star counts.

These estimates suggest that different "candles" may yield different results. In particular it is hard to reconcile the isothermal profile with the steeper declines derived both from simulations and from stellar tracers. The goal of this paper is to extend these works, including turn-off stars. In particular, we propose to recover the halo spatial density by comparing the observed CMD with a synthetic halo population (Monte Carlo generated). Our result is a pure density distribution of stars along the line of sight (no analytical a priori halo density is adopted). Only a posteriori fitting is performed on the recovered density.

Section 2 is dedicated to the sample selection. In Sect. 3 we discuss the methodology. In Sect. 4 the reconstruction of the space density is fitted with a power law, and the robustness of the result is tested against different assumptions on the adopted IMF and metallicity. The possibility of a metallicity gradient is also considered. Our conclusions close the paper.

\section{Data selection}

The OACDF is a multi-color imaging survey mainly for extragalactic studies, acquired with the Wide Field Imager (WFI) at the ESO 2.2-m telescope. This field is located at a high galactic latitude $(l \sim 293, b \sim 50)$, making available a collection of 


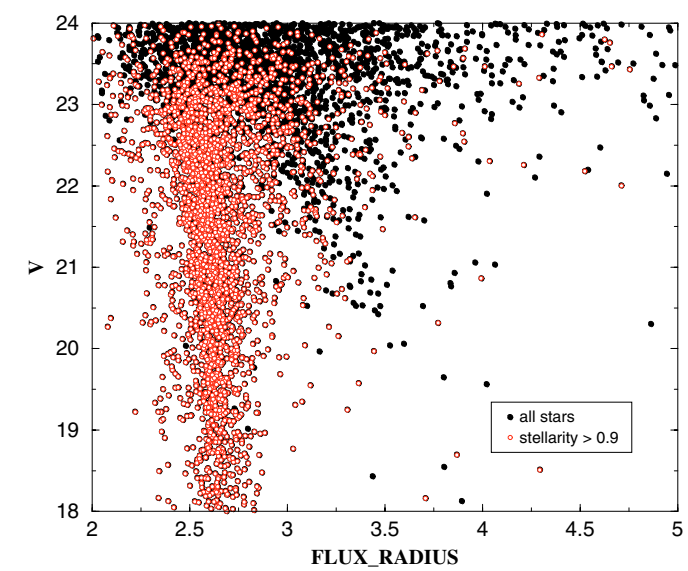

Fig. 1. Visual apparent magnitude versus the sextractor parameter FLUX_RADIUS (two different CLASS_STAR selections are shown: filled circles are all objects, open circles are objects with stellarity larger than 0.9).

halo stars with low contamination of disk and thick-disk stars. The field of view covers an area of about $0.5 \mathrm{deg}^{2}$ in the $B$, $V, R$ optical bands, reaching the following limiting magnitudes: $B_{A B} \sim 25.3, V_{A B} \sim 24.8$, and $R_{A B} \sim 25.1$. The completeness magnitude limit is found at $V_{A B} \sim 24.0$ (for an $S / N 10$ ).

\subsection{Star/galaxy separation}

Contamination by galaxies dominates counts at faint magnitudes. Thus, adequate star/galaxy separation is essential for inferring the Galactic structure. In order to remove the bulk of extra-galactic contaminants, we applied a filtering based on the SExtractor (Bertin \& Arnouts 1996) stellarity parameters (CLASS_STAR) and half-light radius (FLUX_RADIUS). The point-like sources are identified by choosing objects with stellarity higher than 0.9 (simultaneously in $B$ and $V$ filters). Beyond the limit $V=23$, the photometric error in the $V$ filter (see Alcalá et al. 2004) overcomes 0.05 mag, making a clean separation difficult. This finding is evident in Fig. 1, where the stellarity selection is performed in a diagram of $V$ magnitude versus FLUX_RADIUS: selecting stars with high stellarity removes objects with high radius (candidate galaxies). A side effect of this procedure is a loss of very faint stars $(V>23)$. Therefore, we limit our analysis to objects brighter than $V=23$.

The prescription for the stellarity (plus the cut in magnitude) does not ensure the complete removal of all no-stellar objects. Some point-like sources like quasars may be still present. To solve this problem we performed a cut in $B-V$ (rejecting all objects bluer than 0.3 ) with a minimal effect on the stellar population (about 60 objects removed) ${ }^{1}$.

\subsection{Choice of a stellar tracer}

In order to obtain the spatial density of the Galactic halo one must identify stars in a precise evolutionary phase and use them to trace the observed CMD density. The strong requirement on the tracer used to reconstruct the space distribution is that it must be univocally linked to the distance, once metallicity, mass, and age are fixed. In this context, red giants have an important

${ }^{1}$ Halo stars are old and metal poor: assuming the typical $B-V$ color of old globular cluster, we do not expect to find stars bluer than $B-V \approx$ 0.3-0.4 (except a negligible fraction of white dwarfs and HB stars).

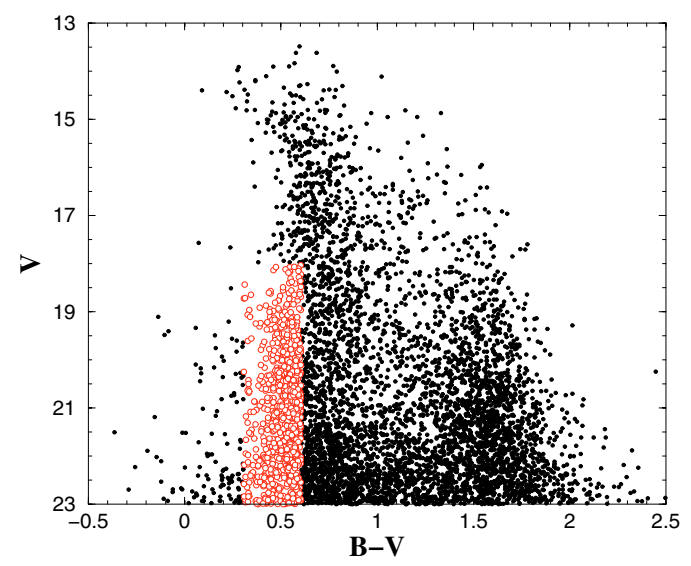

Fig. 2. Color-magnitude diagram of the point-like sources in the OACDF. The turn-off stars discussed in the text are represented with open symbols.

negative effect, because of the degeneracy (in color) with main sequence stars. In contrast, turn-off stars have many advantages:

- They can be univocally selected (definitely bluer than halo red giants);

- They are readily distinguishable from the disk stars in the main sequence (only very young and massive disk stars may have similar colors, but they are rare);

- They are numerous, in fact they outnumber HB stars and red giants by a factor $\gtrsim 100$.

On this basis, we decided to use turn-off stars as tracers of space distribution. To select turn-off stars we defined all objects in our sample with $B-V$ color in the range $0.3-0.6$ (see Fig. 2). The final data contains about 600 stars.

\subsection{Explored distances}

In what follows we present results for stars in the range $18<$ $V<23$. This achieves two goals: the bright limit was set to avoid thick-disk stars, while the faint limit minimizes the contamination of no-stellar sources. Moreover, correlations due to photometric errors are also reduced. These boundaries guarantee a theoretical range between $4 \mathrm{kpc}$ and $60 \mathrm{kpc}^{2}$ (heliocentric).

\section{The model}

A new method for calculating and displaying patterns of Galactic halo stellar density is presented. The recovered distribution $N\left(r_{\odot}\right)$ is the number of stars for heliocentric interval $\delta r_{\odot}$ and solid angle $\omega$. To recover the spatial density behind the observational CMD we need to model a synthetic halo population with various theoretical ingredients. Then the match between theoretical and observational CMDs is quantified (see Cignoni et al. 2006 for details).

Following a Monte Carlo technique, the theoretical CMD is populated with a large number of stars randomly built by means of a set of theoretical ingredients. Ignoring chemical evolution, the synthetic halo population is built near coeval and mono

\footnotetext{
2 A simple population with metallicity $Z=0.001$ and $12 \mathrm{Gyr}$, the turn-off luminosity is near to $M_{V} \sim 4$ (lower metallicities lead to a brighter limit). In this case, a minimum luminosity $V=23$ implies a maximum distance around $60 \mathrm{kpc}$.
} 
metallic. The adopted star formation rate is constant between 10 and 12 Gyr (age estimations for globular clusters indicate values between 11 Gyr, Chaboyer et al. 1998 and 13 Gyr, Hansen et al. 2002). The metallicity is fixed at $[\mathrm{Fe} / \mathrm{H}] \sim-1.6$ (see e.g. Ryan \& Norris 1991) with a Gaussian spread $\left(\sigma_{[\mathrm{Fe} / \mathrm{H}]}=1.0\right)$. The adopted IMF is a power law with a Salpeter index. Once masses, ages, and metallicity are known, colors and magnitudes are obtained by interpolating a library of stellar evolutionary tracks (Pisa Evolutionary Library, Cariulo et al. 2004).

In order to reconstruct the halo structure, this synthetic population is placed at different distances, randomly placing stars in the $j$-th heliocentric interval $\left[d_{\odot, j}, d_{\odot, j}+4 \mathrm{kpc}\right]$. The synthetic diagrams are corrected for reddening and extinction according to the Schlegel et al. (1998) maps (for the OACDF field, a $E(B-V) \approx 0.05$ is adopted). The final product is a base of partial color magnitude diagrams, each one representing the same population but at different distance moduli. Transforming these CMDs in two-dimensional histograms (bin size: $\delta V=0.05$, mag, $\delta(B-V)=0.05 \mathrm{mag})$, we obtain a base in a 2D space ( $V$ versus $B-V$ ) and can write every CMD as a linear combination of these histograms. In numbers, a generic CMD is built as:

$m_{i}=\sum_{j} \alpha_{j} c_{i j}$

where $m_{i}$ is the number of stars in the final CMD in bin $i, \alpha_{j}$ the stellar rate in the distance slab $j$, and $c_{i j}$ the number of stars in the bin $i$ owning to the partial CMD $j$.

The combination of coefficients $\alpha_{j}$ that minimize a Poissonbased likelihood is searched through a simplex algorithm. The uncertainties on the final coefficients are found by using a bootstrap technique. The final result is the distribution of stars along the line of sight in the direction of the OACDF. However, real data are observed in a solid angle, therefore our result is not a density yet. After correcting for the solid angle, the space density as a function of the Galactocentric distance is found by converting from heliocentric distances to Galactocentric distances.

\section{Results}

The recovered spatial distribution is shown in Fig. 3. We have to point out the exact nature of our result. In fact, the OACDF field is not along a galactic radius, but is rather oblique. Thus, comparing our result with simple radial profiles means that we are assuming an axially symmetric Galaxy. Moreover, due to the large uncertainties of the further bins, our analysis is truncated at the galactocentric distance of $40 \mathrm{kpc}$.

A parametrized model of the mass distribution is fitted to the recovered density. The halo is described by a power law $r^{-n}$ ( $r$ galactocentric radius), with axis ratio equals to 1 (round halo).

Different hypotheses for the power law index $n$ (see Fig. 3) are explored through a chi square fitting technique.

Figure 4 illustrates the reduced $\chi_{v}^{2}\left(\chi^{2} /\right.$ degrees of freedom) versus the power law index $n$ : the minimum is placed near $n=$ 2.9 with $\chi_{v}^{2} \approx 1.0$. Although it is difficult to define a confidence interval $^{3}$ for $n$, these results confirm a stellar halo with a decline steeper than an isothermal profile (which is the accepted profile for the dark halo). Indeed, values $n$ lower than 2 give an $\chi_{v}^{2}$ more than 4 times higher than the $n=3$ result.

3 The uncertainties on the recovered densities may hide correlations among bins (incompleteness, external contaminations, and wrong metallicitiy may produce systematic effects; see e.g. Cignoni et al. 2006), making the $\chi^{2}$ probability quite useless.

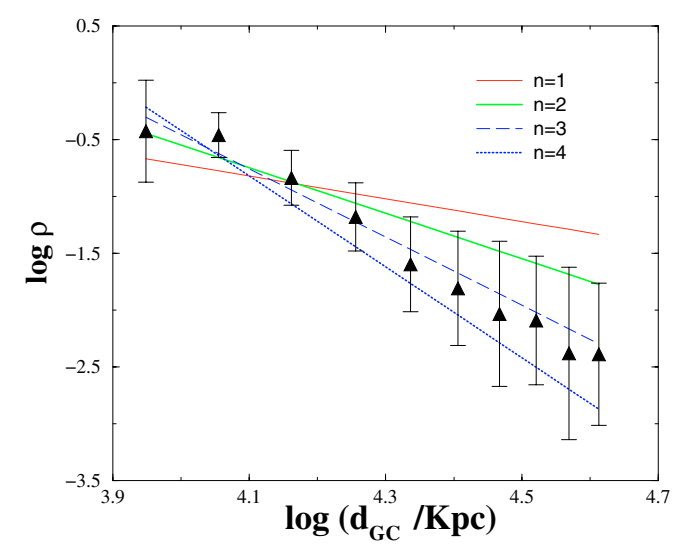

Fig. 3. The recovered density distribution (the density scale is somewhat arbitrary). Error bars are $1 \sigma$. For comparison, different power law densities are also shown (with the labeled exponents).

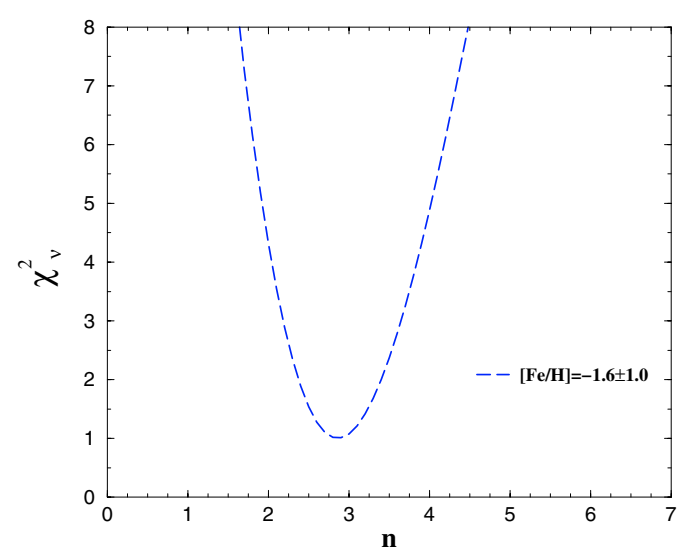

Fig. 4. Reduced $\chi^{2}$ versus the $n$ exponent.

\subsection{Alternative scenarios}

In order to avoid biases due to the assumed halo properties, namely the IMF, the metallicity ${ }^{4}$, and the axis ratio, we have repeated our recovery by using different scenarios.

\subsection{IMFs}

Figure 5 shows the effect of different IMFs. The result is quite independent of the precise IMF $\left(\mathrm{d} N / \mathrm{d} m \propto m^{-s}\right)$ exponent $s$, probably due to the short mass spectrum involved in the halo population. The cuts at $V=23$ and $B-V=0.6$, combined with the typical low metallicities involved in the halo, imply a detectable minimum mass of about $0.6 M_{\odot}$. Then, the age of the halo, which is higher than $10 \mathrm{Gyr}$, implies a maximum mass (for stars still in main sequence) around $0.8 M_{\odot}$. In conclusion, the total mass spectrum is only $\approx 0.2$ solar masses: too short to distinguish between different IMF slopes. Photometric errors, the presence of different metallicities, and low number statistics can largely sweep out an IMF effect.

\subsection{Metallicity effect}

A variation in the metallicity spread has no significant impact (Fig. 6 shows the result for $\Delta[\mathrm{Fe} / \mathrm{H}]=0.3,0.5,1.0$ ). In fact,

\footnotetext{
4 In principle the assumed age is also critical. However, the low masses involved (lower than $0.8 M_{\odot}$ ) imply a weak sensitivity to the age.
} 


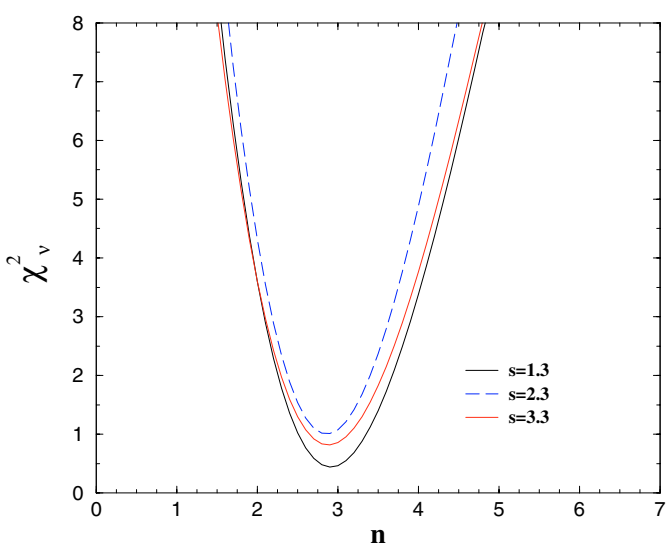

Fig. 5. Reduced $\chi^{2}$ versus index $n$ for different assumed IMF (the explored exponents are labeled).

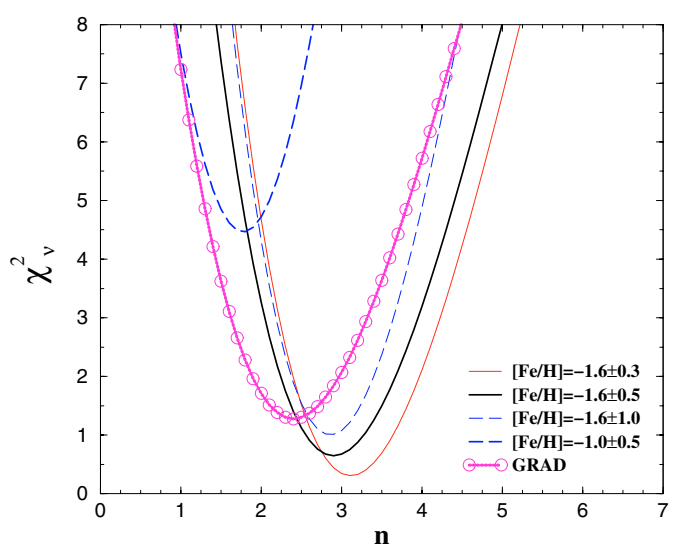

Fig. 6. Reduced $\chi^{2}$ versus index $n$ for several assumed halo metallicities. The metallicity distribution is always a Gaussian with the labeled mean and dispersion; the label "grad" indicates a model with a spatial gradient - see text.

a metallicity spread introduces a blurring on the partial CMDs, which causes an additional random uncertainty on the recovered density. However, this effect is not systematic.

The result is different when the mean metallicity of the model is changed. In particular, increasing the metallicity to $[\mathrm{Fe} / \mathrm{H}]=-1.0$ shifts the most probable $n$ exponent to lower values. The new best value is close to $n=2$, but the relative $\chi_{v}^{2}$ is substantially higher than the previous estimates $(\approx 4.5)$.

Finally, Fig. 6 also shows the resulting $\chi_{v}^{2}$ when an extreme spatial gradient $(0.2$ dex each $4 \mathrm{kpc},[\mathrm{Fe} / \mathrm{H}]=0$ in the Solar position) is adopted (labeled with "grad"). As expected, the adoption of a metallicity gradient has an intermediate effect: the minimum $\chi_{v}^{2}$ is reached for $n \approx 2.3$, yielding an $\chi_{v}^{2}$ value around 1.3 , which is comparable to those resulting from the first models.

\subsection{Axis ratio}

In this section the hypotheses of a round halo is abondoned. Although the axis ratio (AR) is not significantly constrained by a single field, two alternative values are explored $(A R=0.8$ and 0.6). Figure 7 shows the results. Decreasing the AR determines a lower best value for $n$. The extreme choice AR $=0.6 \mathrm{im}$ plies a value $n \approx 2$.2. It is noteworthy that the best $n$, independent

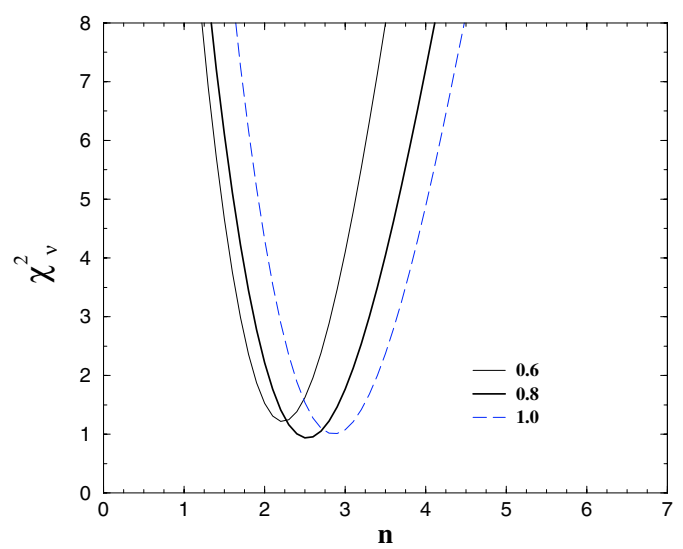

Fig. 7. Reduced $\chi^{2}$ versus index $n$ for several hypothesis on the axis ratio $(\mathrm{AR}=0.6,0.8,1.0)$.

of the precise AR, is characterized by similar chi square values: for a single field, the degeneracy between axis ratio and powerlaw index does not allow the two effects to be disentangled.

\section{Conclusions}

We have combined a star-count model with an updated stellar library to reconstruct the Galactic halo stellar density in the direction of the Capodimonte Deep Field. Our results confirm a steep decline. In particular, when a metallicity of $[\mathrm{Fe} / \mathrm{H}]=-1.6 \pm$ 1.0 is adopted, a power-law index of $n \approx 3$ gives a reasonable fit out to $\approx 40 \mathrm{kpc}$ from the galactic center. This is quite different from the value $n \approx 2$, implied by the flat rotation curve. However, gas and turn off stars may trace different halo regions. First, the measurements of rotation velocity from gas are reliable up to about $20 \mathrm{kpc}$ from the Galactic Center while turn off stars reach $40 \mathrm{kpc}$, suggesting that our exploration is deeper in the outer halo. Second, the OACDF field is high on the Galactic plane while the gas is locked on the plane.

The value $n \approx 3$ is also larger than the Robin et al. (2000) and Siegel et al. (2002) findings, that analyzed several galactic fields (generally shallower than OACDF) using similar stellar tracers. Basically, our study confirms the results obtained with RR Lyrae stars.

A higher metallicity $([\mathrm{Fe} / \mathrm{H}]=-1.0)$ can lower our best value to the isothermal $n \approx 2$, but the corresponding $\chi_{v}^{2}$ is significantly worse. Moreover, the presence of an extreme chemical gradient $\left(\chi_{v}^{2}\right.$ values similar to the $[\mathrm{Fe} / \mathrm{H}]=-1.6 \pm 1.0$ scenario $)$ cannot be ruled out. A lower index $n$ is also obtainable by adopting smaller axis ratios. In these cases, the minimum $\chi_{v}^{2}$ are close to case with round halo ( $\mathrm{AR}=1.0)$, leaving the possibility of a lower index $n(n=2.2$ for $\mathrm{AR}=0.6)$.

This work represents a pilot program for a larger project devoted to the exploration of the VST halo fields (STREGA@VST, see Marconi et al. 2006). The possibility to perform multidirection comparisons will allow us to reconstruct the $3 \mathrm{D}$ pattern of the halo and to disentangle the degeneracy between the axis ratio and the density power law slope. Moreover, the availability of several photometric filters will provide a chance to improve both the comparison between synthetic and observational CMDs and the separation star/galaxies.

Acknowledgements. We are grateful to Steven N. Shore for his interest in and support of this work, and to S. Degl'Innocenti and P. G. Prada Moroni for helpful discussions and for kindly providing specific evolutionary tracks. We thank the OACDF team (http://www.oacn.inaf.it/oacdf-bin/cdfcgi?people) 
for their contribution of data reduction and discussions. Financial support for this study was provided by MIUR, under the scientific project "On the evolution of stellar systems: fundamental step toward the scientific exploitation of VST" (P.I. Massimo Capaccioli).

\section{References}

Alcalá, J. M., Pannella, M., Puddu, E., et al. 2004, A\&A, 428, 339 Avila-Reese, V., Firmani, C., Klypin, A., \& Kravtsov, A. V. 1999, MNRAS, 310, 527

Bertin, E., \& Arnouts, S. 1996, A\&AS, 117, 393

Cariulo, P., Degl'Innocenti, S., \& Castellani, V. 2004, A\&A, 421, 1121

Cignoni, M., Degl'Innocenti, S., Prada Moroni, P. G., \& Shore, S. N. 2006, A\&A, 459, 783

Chaboyer, B., Demarque, P., Kernan, P. J., \& Krauss, L. M. 1998, ApJ, 494, 96

Eggen, O. J., Lynden-Bell, D., \& Sandage, A. R. 1962, ApJ, 136, 748
Gould, A., Flynn, C., \& Bahcall, J. N. 1998, ApJ, 503, 798

Hansen, B. M. S., Brewer, J., Fahman, G. G., et al. 2002, ApJ, 574, 155

Ivezic, Z., Lupton, R., Schlegel, D., et al. 2004, Satellite and Tidal Streams, ed. F. Prada, D. Martinez-Delgado, \& T. Mahoney (San Francisco: ASP), ASP Conf. Ser., 327, 104

Marconi, M., Musella, I., Ripepi, V., et al. 2006, Mem. Soc. Astron. It. Supp., 9, 253

Nissen, P. E., \& Schuster, W. J. 1997, A\&A, 326, 751

Prada, F., Vitvitska, M., Klypin, A., et al. 2003, ApJ, 598, 260

Robin, A. C., Reylé, C., \& Crézé, M. 2000, A\&A, 359, 103

Schlegel, D. J., Finkbeiner, D. P., \& Davis, M. 1998, ApJ, 500, 525

Searle, L., \& Zinn, R. 1978, ApJ, 225, 357

Siegel, M. H., Majewski, S. R., Reid, I. N., \& Thompson, I. B. 2002, ApJ, 578, 151

Ryan, S., \& Norris, J. 1991, AJ, 101, 1865

Sommer-Larsen, J., \& Zhen, C. 1990, MNRAS, 242, 10

Vivas, A. K., \& Zinn, R. 2003, Mem. Soc. Astron. It., 74, 928 